\title{
Magnesium interatomic potential for simulating plasticity and fracture phenomena
}

\author{
Z Wu $\mathbf{u}^{1,2} \uparrow, \mathbf{M}$ F Francis ${ }^{1,3}$ and W A Curtin ${ }^{1}$ \\ ${ }^{1}$ Institute of Mechanical Engineering, École Polytechnique Fédérale de Lausanne, Lausanne \\ CH-1015, Switzerland \\ 2 Institute of High Performance Computing, 1 Fusionopolis Way, \#16-16, Connexis, \\ Singapore 138632, Singapore \\ ${ }^{3}$ Brown University, Providence, Rhode Island 02912, United States \\ E-mail: zhaoxuan.wu@epfl.ch
}

\begin{abstract}
.
Magnesium has multiple dislocation and twinning systems with starkly different properties, which make its plastic deformation strongly anisotropic and highly complex. Existing empirical interatomic potentials fail to capture the full scope of these properties, making current molecular statics and dynamics simulation results of limited quantitative and predictive use. Here, based on the work by Kim et al, a new modified embedded-atom method potential for magnesium is introduced and rigorously validated against existing $a b$ initio, continuum theory and experimental results. The new potential satisfactorily reproduces all the necessary mechanical properties for plastic deformation, including the various generalized stacking fault energy surfaces, dislocations core structures, Peierls stresses, surface energies, and basal plane cohesive strength. The capability of this potential to accurately describe all the important slip systems and fracture behavior makes it valuable for future realistic atomistic studies of general magnesium deformation and failure problems.
\end{abstract}

PACS numbers: 61.72.Lk, 62.20.F-, 62.20.mt

Keywords: Magnesium; Dislocations; Fracture; Empirical Potentials; Molecular Dynamics Simulations

Submitted to: Modelling Simul. Mater. Sci. Eng.

$\ddagger$ Author to whom any correspondence should be addressed. 
This is an author-created, un-copyedited version of an article accepted for publication/published in Modelling and Simulation in Materials Science and Engineering. IOP Publishing Ltd is not responsible for any errors or omissions in this version of the manuscript or any version derived from it. The Version of Record is available online at http://dx.doi.org/10.1088/0965-0393/23/1/015004. Citation details: Wu, Z.; Francis, M. F.; Curtin, W. A. Model. Simul. Mater. Sci. Eng. 2015, 23 (1), 15004.

\section{Introduction}

Magnesium (Mg) and its alloys possess extraordinary mechanical and physical properties. They have very high specific strength, good machinability, are relatively easy to recycle, and $\mathrm{Mg}$ is abundant in the Earth's crust. Mg alloys are thus attractive structural materials in a wide range of applications in the automotive and aerospace industry, and in energy and consumer products [1]. However, the adoption of Mg-based alloys is significantly inhibited by their limited deformability at low and moderate temperatures. In fact, pure $\mathrm{Mg}$ has little ductility and very poor formability at room temperature. Low ductility makes the material intolerant to microstructural defects and thus susceptible to brittle fracture/catastrophic failure, while poor formability makes $\mathrm{Mg}$ incompatible with established forming techniques and difficult to process.

Generally, the low deformability of $\mathrm{Mg}$ can be attributed to its hexagonal close-packed (hcp) crystal structure. The hcp structure has one close-packed basal plane offering two independent dislocation slip systems with glide at low stresses, but these are insufficient to satisfy the von Mises criterion [2] for general plastic strain. In order to accommodate $\boldsymbol{c}$-axis strain, activation of non-basal slip modes is necessary. However, slip on non-basal planes is complex, requiring much higher stresses than for basal slip and often dependent on loading conditions. Furthermore, the slip is accomplished by dislocations with relatively larger Burgers vectors on multiple slip planes or by twinning accompanied by atomic shuffling processes. These very dissimilar properties among the different slip modes make the plastic deformation of $\mathrm{Mg}$ strongly anisotropic and highly complex.

Given the vast potential of Mg-based alloys, many experimental studies [3, 4, 5, 6, 7, 8] have been carried out to shed light on the deformation process of $\mathrm{Mg}$. However, because of the wide ranges of time and length scales involved and electron transparency requirements, direct observations of dislocation structures and key atomistic processes remain challenging. Atomistic simulations are thus helpful to complement experiments and provide critical insights into the deformation process of materials [9]. Among them, molecular statics (MS) and molecular dynamics (MD) simulations can be particularly useful in revealing atomicscale deformation mechanisms if the underlying empirical potential correctly reproduces the relevant material properties. For $\mathrm{Mg}$, capturing the multiple slip systems with disparate properties has proven difficult for all potentials developed to date. Predictions of dislocation properties using empirical potentials can be quite different from those obtained in experiments and or via first-principles density function theory (DFT) calculations. The embedded-atom method (EAM [10]) potentials (Liu [11] and Sun [12]) can give unrealistic generalized stacking fault (GSF) energy [13, 14], Peierls barriers [13], and $\langle\boldsymbol{c}+\boldsymbol{a}\rangle$ dislocation core structures [15]. The EAM potential developed by Sun et al [12] also gives unstable $\{10 \overline{1} 2\}\langle\overline{1} 011\rangle$ twinning dislocation, and unrealistic twin interface structure and basal surface energy. While each potential can provide good predictions for some dislocations and/or some properties, there is no single EAM potential that is adequate for representing all the necessary properties to study $\mathrm{Mg}$ deformation under general plastic strain or in polycrystal structures.

Recently, Kim et al developed a second nearest neighbor modified embedded-atom 
This is an author-created, un-copyedited version of an article accepted for publication/published in Modelling and Simulation in Materials Science and Engineering. IOP Publishing Ltd is not responsible for any errors or omissions in this version of the manuscript or any version derived from it. The Version of Record is available online at http://dx.doi.org/10.1088/0965-0393/23/1/015004. Citation details: Wu, Z.; Francis, M. F.; Curtin, W. A. Model. Simul. Mater. Sci. Eng. 2015, 23 (1), 15004.

method (MEAM [16, 17]) potential for pure Mg and the Mg-Al system [18]. The potential for pure $\mathrm{Mg}$ (labelled the Kim potential hereafter) was shown to reproduce the $\langle\boldsymbol{c}+\boldsymbol{a}\rangle$ dislocation core structures on pyramidal II plane comparable to those of DFT calculations [15]. This is a major success. However, the Kim potential has not been widely tested, validated, or used for other dislocation core structures or twin interfaces. Moreover, in using the Kim potential to simulate basal plane crack propagation, we have found that the Kim potential exhibits strong and unphysical "lattice trapping", making it unsuitable for fracture studies. While having some promise, the existing Kim potential still requires improvements and validation.

In this work, we present a newly parametrized $\mathrm{Mg}$ potential (labelled the MEAM potential hereafter) that rectifies the problems of the original Kim potential with respect to basal fracture while retaining many attractive properties of the Kim potential. In addition, we make extensive quantitative comparisons between the MEAM potential and available DFT results for predictions of the basal cohesive separation and cohesive strength, the GSF energy, and the dislocation core structures and Peierls barriers for basal, prismatic, pyramidal I and II, and tensile twinning slip modes. We also compare the fracture behavior of the MEAM potential with continuum theories for cleavage and dislocation emission criteria for two different crystallographic orientations. Through this extensive testing, we show that the new MEAM potential reproduces all dislocation and fracture properties consistent with available DFT results and continuum theory. We thus recommend that this new MEAM potential for $\mathrm{Mg}$ be the sole interatomic potential used for all future studies of general $\mathrm{Mg}$ deformation and failure problems in atomistic simulations.

The remainder of this paper is organized as follows. In Section 2, we describe our simulation methods and geometries. In Section 3, we present the detailed changes made to the Kim potential and the resulting properties. In Sections 4 and 5, we show the results of GSF energy calculations and dislocation properties. Results on fracture tests are presented in Section 6, followed by discussion and conclusions in Section 7.

\section{Simulation methods and geometries}

Figure 1 (a-e) shows the Mg slip systems chosen in this study. In all the simulation geometries, the slip plane is the $x-z$ plane with the $z$ axis parallel to the dislocation line and the $y$-axis is normal to the slip plane. We examine both edge and screw dislocations on all the five slip planes shown in figure 1. Periodic boundary conditions are used in the dislocation line $(z)$ direction while the surfaces normal to the $x$ and $y$ directions are traction free. The dimensions in the $x$ and $y$ directions are $\sim 100 \mathrm{~nm}$ each and $\sim 2 \mathrm{~nm}$ in the $z$ direction. All dislocations are created at the center of the simulation cell using the anisotropic elastic Volterra displacement field [2]. Dislocation core structures are visualized using both the component of the Nye tensor plot [19] and differential displacement (DD) plot [20]. These results are compared directly with similar data obtained from available DFT studies in the literature.

In the calculations of the Peierls stresses, we consider three temperatures, $T=0 \mathrm{~K}$, $77 \mathrm{~K}$ and $300 \mathrm{~K}$, the latter two enabling better comparison with experimental results. In simulations of $T=0 \mathrm{~K}$, forces are applied to those atoms within the potential cut-off distance 
from the upper and lower $y$ surfaces to create the desired simple shear stress, followed by energy minimization. Such forces are increased gradually (corresponding to less than 5\% of the final stress at each increment) until the dislocation start to move. In simulations of finite temperatures, atom positions are first scaled from $T=0 \mathrm{~K}$ using the finite-temperature lattice parameters, followed by initializing the atom velocities using a Gaussian distribution. To damp out excessive elastic waves/stress variations, all systems are first equilibrated with a Langevin thermostat for 200 ps, followed by a Nosé-Hoover thermo/barostats [21, 22, 23, 24] to maintain constant temperature and zero normal stress in the periodic direction for another 100 ps. The above procedure can equilibrate the systems so that the amplitudes of all stress variations are in the range of several $\mathrm{MPa}$, in agreement with the range of thermodynamic expectation at room temperature. We emphasize that this procedure of equilibration is crucial for preparing systems at finite temperatures and reliable measurement of the Peierls stresses. Following equilibration, constant temperature and zero normal stress in the periodic direction are maintained by using a Nosé-Hoover thermo/barostats, while forces are applied as in the $T$ $=0 \mathrm{~K}$ simulations and are increased every $10 \mathrm{ps}$ with a magnitude corresponding to less than $5 \%$ of the final stress.

figure_1.pdf

Figure 1. Common slip systems in Mg: (a) basal (b) prismatic (c) pyramidal I (d) pyramidal II and (e) tension twin. (f-g) Simulation cells and orientations for two basal plane cracks.

The dislocation energy can be estimated by measuring the excessive energy per unit length within a cylindrical region of radius $r$ centered at the dislocation core as a function of radius $r$, i.e.,

$$
E(r)=E\left(r_{c}\right)+A \ln \left(r / r_{c}\right)
$$

where $A$ is a known prefactor associated with the anisotropic material elastic constants and $r_{c}$ is a core radius. The energy difference between two dislocations is therefore

$$
\Delta E(r)=E^{(1)}(r)-E^{(2)}(r), \text { for } r>r_{c}
$$


This is an author-created, un-copyedited version of an article accepted for publication/published in Modelling and Simulation in Materials Science and Engineering. IOP Publishing Ltd is not responsible for any errors or omissions in this version of the manuscript or any version derived from it. The Version of Record is available online at http://dx.doi.org/10.1088/0965-0393/23/1/015004. Citation details: Wu, Z.; Francis, M. F.; Curtin, W. A. Model. Simul. Mater. Sci. Eng. 2015, 23 (1), 15004.

Since screw dislocations with the same Burgers vector have the same crystal orientation, they share the same long-range elastic field (including the image forces) and thus only differ in the dislocation core structure and core energy. Equation (2) thus gives directly the core energy difference. For edge dislocations, the core energy difference can be estimated at $r \rightarrow r_{c}$ or by subtracting the computed elastic energy from equation (1).

In calculations of the GSF energy, periodic boundary conditions are used in the $x$ and $z$ directions while the $y$ surfaces are free with height larger than 100 times the interplanar spacing along $y$. To calculate the GSF energy, we follow the standard procedure in which the upper half of the crystal is rigidly shifted in the $x-z$ plane followed by energy minimization with all atoms fixed in the $x$ and $z$ directions and free to relax in the $y$ direction.

In the benchmark of fracture properties, we examine two semi-infinite cracks with different crystallographic orientations: (0001)[1010] for orientation I and (0001)[12 10 ] for orientation II as shown in figure 1 (f) and (g). Orientation I favors $\langle\boldsymbol{c}+\boldsymbol{a}\rangle$ dislocation nucleation on pyramidal I planes and potentially twinning dislocation nucleation while orientation II favors $\langle\boldsymbol{c}+\boldsymbol{a}\rangle$ dislocation nucleation on pyramidal II planes. The two simulation cells have dimensions $(x \times y \times z)$ of $2388 \AA \times 2290 \AA \times 19 \AA$ and $2405 \AA \times 2290 \AA \times 17 \AA$, respectively. Atoms within $\sim 2 r_{c}$ from the boundary are set as outer boundary atoms. To load the crack, the anisotropic elastic displacement field of mode I crack tip stress intensity factor $K_{I}$ is used for the initial atom positions [25]. Relaxation of the system to minimize the total energy is then performed while holding the outer boundary atoms fixed at their anisotropic elastic displacements.

MS and MD simulations are performed using the Large-scale Atomic/Molecular Massively Parallel Simulator (LAMMPS) [26]. DFT calculations are performed using the Vienna Ab-initio Simulation Package (VASP) [27, 28] with a plane-wave spin free basis set and the projector augmented wave (PAW) [29] method. A Monkhorst-Pack [30] automatic $\mathrm{k}$-mesh generation scheme is used with 40 subdivisions along each reciprocal lattice lattice vector; the k-mesh is shifted with respect to the origin by 0.5 reciprocal lattice vectors. The Perdew-Wang-91 (PW91) [31, 32] generalized gradient approximation (GGA) functionals are used for exchange-correlation potential. All energy minimizations are carried out using a conjugate gradient algorithm.

\section{MEAM Potentials}

In simulations of basal crack propagation using the Kim potential, we have found that atomically-sharp cracks on basal planes neither advance nor emit dislocations even under loads that are several times larger than the critical stress intensity factor for Griffith cleavage. This "lattice trapping" of cracks is well-established theoretically [33, 34] and in atomistic simulations [35, 36], and large lattice trapping is unexpected for most metallic systems. In order to identify the origin of the lattice trapping, we first computed the cohesive energy $E_{0}$ of crystalline $\mathrm{Mg}$ as a function of the lattice parameter $a$ with $c / a$ fixed. We then computed the decohesion or separation energy $\gamma_{s}(d)$ and cohesive traction-separation law $\sigma_{c}(d)$ as a function of the separation $d$ normal to the basal plane. Figure 2 shows the results. For the Kim 
This is an author-created, un-copyedited version of an article accepted for publication/published in Modelling and Simulation in Materials Science and Engineering. IOP Publishing Ltd is not responsible for any errors or omissions in this version of the manuscript or any version derived from it. The Version of Record is available online at http://dx.doi.org/10.1088/0965-0393/23/1/015004. Citation details: Wu, Z.;

Francis, M. F.; Curtin, W. A. Model. Simul. Mater. Sci. Eng. 2015, 23 (1), 15004.

potential, the cohesive energy $E_{0}$ as a function of $a$, although smooth, changes rapidly near the cut-off distance of $4.8 \AA$ used in the potential. This rapid change results in unphysically large forces (the negative of the gradient of energy) on atom separations approaching the cutoff distance. This is also seen in the decohesion behavior $\gamma_{s}$ v.s. $d$ as shown in figure 2 (b). There is a rapid change in $\gamma_{s}$ as $d$ approaches the cut-off distance, and a corresponding spurious force (note that $\sigma_{c}$ near the cut-off distance far exceeds the upper range in the plot). In contrast, the DFT results show that (i) both $\gamma_{s}$ and its derivative are continuous and smooth functions of $d$ and (ii) substantial atomic interactions persist well beyond $d>1.8 \AA$, i.e., well beyond the cut-off distance of $4.8 \AA$ used in the Kim potential.

figure_2.pdf

Figure 2. (a) Cohesive energy as a function of lattice size. (b) Decohesion energy per unit area and cohesive traction as a function of basal planar separation.

The fracture behavior of the Kim potential is thus unphysical. Our results indicate that a large cut-off distance is necessary in order to both maintain the characteristics of the Kim potential but also have realistic atomic forces near the cut-off distance. We note that the cut-off distance of the Kim potential is chosen to be between the second and third nearest neighbors so that the second nearest neighbor interactions are directly included. Scanning the pair-distribution function of hcp Mg together with the DFT calculations shown in figure 2 (b) suggests that the ideal cut-off distance could be between the fourth and fifth neighbors at $5.875 \AA$ or sixth and seventh neighbors at $7.5 \AA$. We therefore refitted the Kim potential with these two cut-off distances following the same procedures as described in the work of Kim et al [18]. The main purpose of extending the cut-off distance is to have a smoother change of the cohesive energy $E_{0}$ and surface formation energy $\gamma_{s}$ near the cut-off distance. Therefore, we retain the values of the screening parameters $C_{\min }$ and $C_{\text {max }}$ used in the Kim potential but extend both the radial cut-off distance $r_{c}$ and radial cut-off region $\Delta r$. Retaining the screening means that the effects of extending the cut-off distance on many physical bulk properties of the potential are small, as we show below. We carried out extensive testing of both cut-off distances and found that no appreciable difference can be observed in dislocation core structures and fracture behavior. There is a higher computational cost with the larger cut-off distance, however, with the perfect hcp lattice having 68 neighbor atoms using the larger cut-off as compared to 38 atoms for the shorter cut-off. Therefore, we focus here on the 
This is an author-created, un-copyedited version of an article accepted for publication/published in Modelling and Simulation in Materials Science and Engineering. IOP Publishing Ltd is not responsible for any errors or omissions in this version of the manuscript or any version derived from it. The Version of Record is available online at http://dx.doi.org/10.1088/0965-0393/23/1/015004. Citation details: Wu, Z.;

Francis, M. F.; Curtin, W. A. Model. Simul. Mater. Sci. Eng. 2015, 23 (1), 15004.

Magnesium interatomic potential for simulating plasticity and fracture phenomena

shorter cut-off distance of $5.875 \AA$ for both computational efficiency and satisfactory accuracy. In fitting the remaining MEAM potential parameters, emphases are placed on reproducing the experimental/ab initio lattice parameters (particularly the $c / a$ ratio, which is very important for twinning), the basal plane surface energy, and the elastic constants, consistent with our primary interest in studying plastic deformation and fracture of $\mathrm{Mg}$.

Table 1 shows the parameter values for the new MEAM potential together with the values previously used in the Kim potential. Figure 2 (a) and (b) show the predictions for $E_{c}$ v.s. $a$ and $\gamma_{s}$ v.s. $d$ using the MEAM potential, in which it is evident that the MEAM potential retains the attractive properties of the Kim potential while also reproducing the DFT results. Table 2 shows the calculated material properties of the MEAM potential in comparison with experimental/ab initio data and the Kim potential. Overall, these baseline material properties of the MEAM potential are in good agreement with experimental/ab initio results, as well as with the results of the Kim potential. In particular, the lattice parameters $a$ and $c / a$ of the MEAM potential match ideally with experimental values at room temperature. This could be an important property affecting twinning deformation of $\mathrm{Mg}$ [37]. We present further test results on this new MEAM potential in the following sections.

\begin{tabular}{ccccccccccccccc}
\hline & $E_{c}$ & $r_{e}$ & $B$ & $A$ & $\beta^{(0)}$ & $\beta^{(1)}$ & $\beta^{(2)}$ & $\beta^{(3)}$ & $t^{(1)}$ & $t^{(2)}$ & $t^{(3)}$ & $C_{\min }$ & $C_{\max }$ & $d$ \\
\hline & & & & & & & & & & & & & & \\
MEAM & 1.51 & 3.19 & 0.37 & 0.52 & 2.00 & 1.30 & 1.30 & 1.00 & 5.55 & 3.00 & -7.40 & 0.49 & 2.90 & 0.00 \\
$\operatorname{Kim}$ & 1.55 & 3.20 & 0.37 & 0.52 & 2.30 & 1.00 & 3.00 & 1.00 & 9.00 & -2.00 & -9.5 & 0.49 & 2.90 & 0.00 \\
\hline
\end{tabular}

Table 1. Parameters for the MEAM potential. Parameters for the Kim [18] potential is also listed for comparison. The units of $E_{c}, r_{e}$ and $B$ are $\mathrm{eV}, \AA$ and $10^{12} \mathrm{dyne} / \mathrm{cm}^{2}$, respectively.

\section{Generalized stacking fault energy}

The GSF energy is an important property that can dictate the slip behavior of crystalline materials. The unstable stacking fault energy along the slip direction can also affect dislocation emission at crack tips. Therefore, accurate GSF energies are necessary precursors for obtaining correct plastic deformation behavior. For Mg, the GSF energies are particularly important in order to accurately reproduce the strongly anisotropic slip behavior. We thus first examine the GSF energy $(\gamma)$ surface on the basal, prismatic, pyramidal I, and pyramidal II planes, as shown in figure 3. Comparing the GSF energy surfaces shows that the basal plane has the lowest stacking fault energy along the dislocation slip direction, followed by the prismatic plane. The minimum energy path (MEP) on the prismatic plane is along the $\langle\boldsymbol{a}\rangle$ direction, consistent with DFT calculations [14] and in contrast with previous study [13, 14] using EAM potentials (Liu [11] and Sun [12]) where unusual MEPs on prismatic plane were found.

On the pyramidal I and II planes, the stacking fault energies along the $\langle\boldsymbol{c}+\boldsymbol{a}\rangle$ dislocation slip direction are similar, and substantially higher than those on basal and prismatic planes. This suggests that nucleation of $\langle\boldsymbol{c}+\boldsymbol{a}\rangle$ dislocations on these pyramidal planes is much more 
This is an author-created, un-copyedited version of an article accepted for publication/published in Modelling and Simulation in Materials Science and Engineering. IOP Publishing Ltd is not responsible for any errors or omissions in this version of the manuscript or any version derived from it. The Version of Record is available online at http://dx.doi.org/10.1088/0965-0393/23/1/015004. Citation details: Wu, Z.;

Francis, M. F.; Curtin, W. A. Model. Simul. Mater. Sci. Eng. 2015, 23 (1), 15004.

Magnesium interatomic potential for simulating plasticity and fracture phenomena

\begin{tabular}{cccc}
\hline $\mathrm{Mg}$ & Exp/ab initio & MEAM & Kim \\
\hline$a(\AA)$ & 3.187 & 3.187 & 3.210 \\
$a(300 \mathrm{~K} \AA)$ & $3.209^{a}$ & 3.210 & 3.233 \\
$c / a$ & 1.631 & 1.623 & 1.620 \\
$c / a(300 \mathrm{~K})$ & $1.624^{a}$ & 1.624 & 1.621 \\
$E_{c}(\mathrm{eV} / \mathrm{atom})$ & $-1.51^{b}$ & -1.51 & -1.55 \\
& & & \\
$C_{11}(\mathrm{GPa})$ & $63.5^{c}$ & 64.3 & 62.9 \\
$C_{12}(\mathrm{GPa})$ & $25.9^{c}$ & 25.5 & 26.0 \\
$C_{13}(\mathrm{GPa})$ & $21.7^{c}$ & 20.3 & 21.2 \\
$C_{33}(\mathrm{GPa})$ & $66.5^{c}$ & 70.9 & 69.7 \\
$C_{44}(\mathrm{GPa})$ & $18.4^{c}$ & 18.0 & 17.1 \\
& & & \\
$\gamma_{t w}(10 \overline{1} 2)\left(\mathrm{mJ} / \mathrm{m}^{2}\right)$ & $118^{d}$ & 148 & 143 \\
$\gamma_{s f}(\mathrm{I} 2)\left(\mathrm{mJ} / \mathrm{m}^{2}\right)$ & $22^{d}, 33^{e}$ & 22.5 & 30 \\
$\gamma_{u s}(\mathrm{I} 2)\left(\mathrm{mJ} / \mathrm{m}^{2}\right)$ & $92^{e}, 108^{f}$ & 125 & 125 \\
$\gamma_{u s}(\mathrm{Prism})\left(\mathrm{mJ} / \mathrm{m}^{2}\right)$ & $218^{g}$ & 220 & 215 \\
& & & \\
$\gamma_{s}(0001)\left(\mathrm{mJ} / \mathrm{m}^{2}\right)$ & $526^{h}, 550^{h}, 620^{h}, 575$ & 568 & 645 \\
$\gamma_{s}(10 \overline{1} 0)\left(\mathrm{mJ} / \mathrm{m}^{2}\right)$ & $856^{h}, 880^{h}, 965^{h}$ & 582 & 839 \\
$\gamma_{s}(11 \overline{2} 0)\left(\mathrm{mJ} / \mathrm{m}^{2}\right)$ & $716^{h}, 737^{h}, 819^{h}$ & 651 & 747 \\
$\gamma_{s}(1 \overline{1} 01)\left(\mathrm{mJ} / \mathrm{m}^{2}\right)$ & $639^{h}, 657^{h}, 726^{h}$ & 767 & 790 \\
$\gamma_{s}(11 \overline{2} 2)\left(\mathrm{mJ} / \mathrm{m}^{2}\right)$ & & 647 & 864 \\
$\gamma_{s}(10 \overline{1} 2)\left(\mathrm{mJ} / \mathrm{m}^{2}\right)$ & $764^{h}, 780^{h}, 856^{h}$ & 661 & 807 \\
\hline
\end{tabular}

Table 2. Pure Mg material properties from experiments/ab initio calculations and reproduced by empirical potentials.

${ }^{a}$ Reference [38]

${ }^{b}$ Reference [39]

${ }^{c}$ Reference [40]

${ }^{d}$ Reference [41]

${ }^{e}$ Reference [42]

${ }^{f}$ Reference [43]

${ }^{g}$ Reference [13]

${ }^{h}$ Reference [44]

difficult than nucleation of $\langle\boldsymbol{a}\rangle$ dislocations on basal and prismatic planes. The MEP on the pyramidal II plane follows the $\langle\boldsymbol{c}+\boldsymbol{a}\rangle$ direction while on the pyramidal I plane, the MEP is more complex. Overall, these GSF energy surfaces are consistent with available DFT results $[13,14,45]$.

Figure 4 shows the GSF energy curves along specific directions for the basal, prismatic, pyramidal I, and pyramidal II planes. In all the cases, the current MEAM and Kim potentials show nearly identical results. In addition, both potentials show reasonable, quantitative agreement with DFT calculations. Most importantly, the unstable and stable stacking fault energies, $\gamma_{u s}$ and $\gamma_{s f}$, are in good agreement with DFT in terms of their magnitudes and locations along the slip directions. For the basal plane, all three models show a deep energy minimum corresponding to the I2 stable stacking fault (SF), as dictated by its local face- 
figure_3.pdf

Figure 3. Generalized stacking fault energy on (a) basal (b) prismatic (c) pyramidal I (d) pyramidal II planes. In each sub figure, the white, dotted line marks the minimum energy path in the $\gamma$ surface.

centered cubic symmetry. This also suggests a strong tendency for dislocation dissociation on this plane. In contrast, for the prismatic plane, no stable SF is seen in DFT while only a shallow energy minimum at half of the slip vector is predicted by the empirical potentials. Thus undissociated dislocation cores are likely in the prismatic planes. Both pyramidal I and II planes show stable SF and unstable SF energies that are much higher than those for basal and prismatic planes. Despite their high stable SF energies, dislocation dissociation is likely due to the large $\langle\boldsymbol{c}+\boldsymbol{a}\rangle$ Burgers vector, since dissociation into partials can substantially lower the elastic energy of the dislocation in accordance with Frank's rule [2]. In the case of the pyramidal II plane, both potentials show a higher energy $(\sim 20 \%)$ than the DFT values, as noted in a previous study of the Kim potential [15].

\section{Dislocation Properties}

The previous sections show that both the MEAM and Kim potentials reproduce the physical properties and GSF energies of $\mathrm{Mg}$ in quite good agreement with experimental/ab initio 
This is an author-created, un-copyedited version of an article accepted for publication/published in Modelling and Simulation in Materials Science and Engineering. IOP Publishing Ltd is not responsible for any errors or omissions in this version of the manuscript or any version derived from it. The Version of Record is available online at http://dx.doi.org/10.1088/0965-0393/23/1/015004. Citation details: Wu, Z.; Francis, M. F.; Curtin, W. A. Model. Simul. Mater. Sci. Eng. 2015, 23 (1), 15004.

figure_4.pdf

Figure 4. Generalized stacking fault energy curves for (a) basal (b) prismatic (c) pyramidal I (d) pyramidal II planes. DFT data are extracted from Refs [43], [13], [45], and [15], respectively. In sub figure (a), the generalized stacking fault energy curve is identical for shifts along the leading and trailing partial dislocation Burgers vectors and is drawn only for the leading partial. In sub figure (c), the generalized stacking fault energy curve along minimum energy path (MEP) is drawn by projecting it along the $\langle\mathbf{c}+\mathbf{a}\rangle$ Burgers vector.

results. To ensure that the potential is suitable for study of general plastic deformation, we now turn our focus to the prediction of dislocation properties, including the dislocation core structures and Peierls barriers. Because the MEAM potential exhibits no appreciable differences from the Kim potential, we only show the results of the MEAM potential in comparison with available DFT results.

We first examine the $\langle\boldsymbol{a}\rangle$ dislocations on basal planes. Figure 5 shows the basal edge and screw dislocation core structures. In each case, the basal dislocation dissociates into two partial dislocations, each with both edge and screw components as shown via the Nye tensor contours. This dissociation is consistent with the basal plane GSF energy surface shown in figure 3. Edge dislocations have wider core separation than screw dislocations (see Table 3), as expected. Both the edge and screw dislocation core structures of the MEAM potential match well with the corresponding DFT structures, except for the core separation of the edge dislocation. The MEAM edge dislocation shows a wider core separation than the DFT core, which may be partially due to the lower basal plane stacking fault energy in the MEAM 
This is an author-created, un-copyedited version of an article accepted for publication/published in Modelling and Simulation in Materials Science and Engineering. IOP Publishing Ltd is not responsible for any errors or omissions in this version of the manuscript or any version derived from it. The Version of Record is available online at http://dx.doi.org/10.1088/0965-0393/23/1/015004. Citation details: Wu, Z.;

Francis, M. F.; Curtin, W. A. Model. Simul. Mater. Sci. Eng. 2015, 23 (1), 15004.

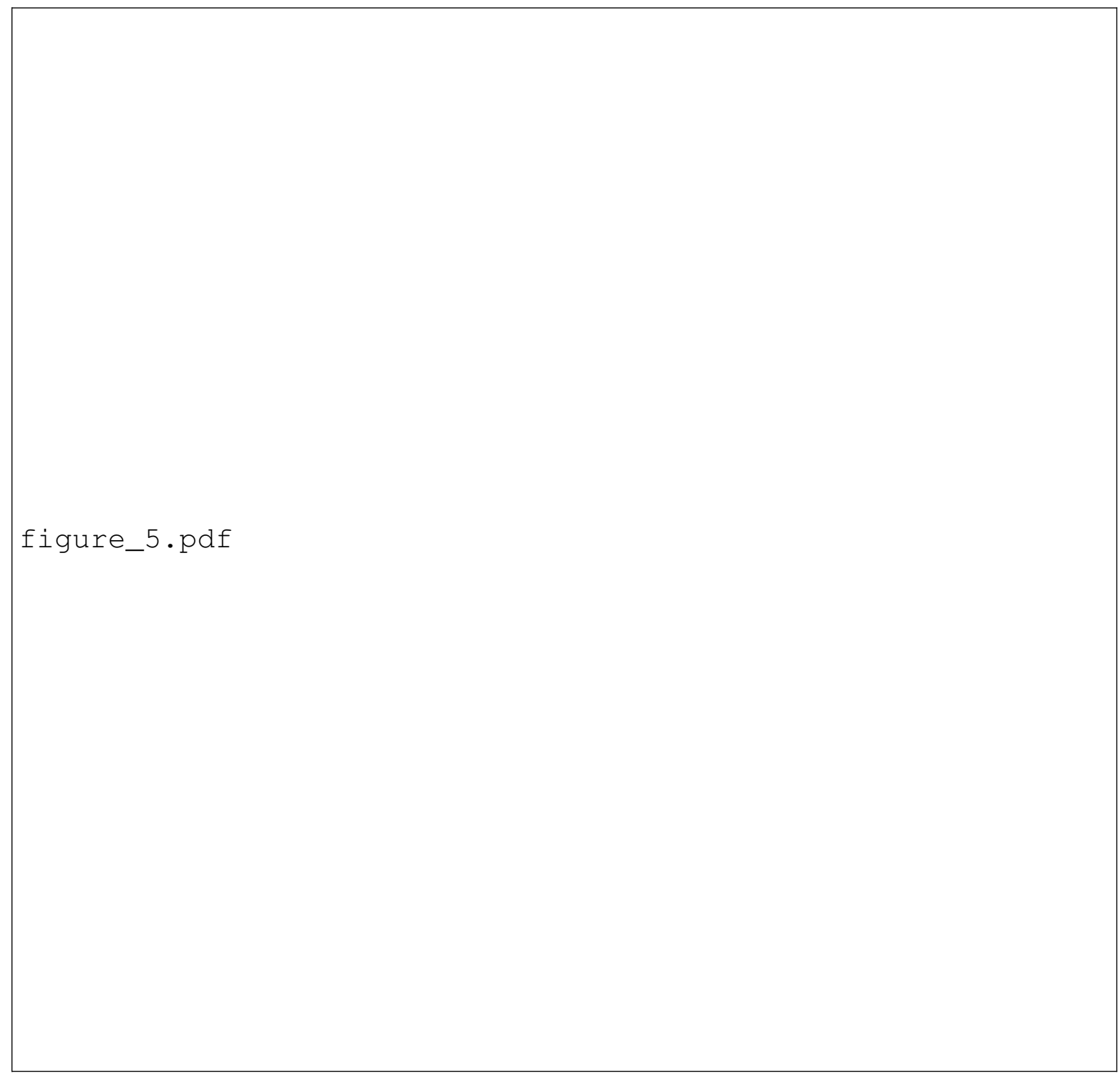

Figure 5. Basal $\langle\boldsymbol{a}\rangle$ edge and screw dislocation core structures as visualized by the component of the Nye tensor and differential displacement plots. DFT results are replotted based on atom positions in Ref [13].

potential than those obtained in most DFT calculations.

\begin{tabular}{lllllllll}
\hline & \multicolumn{8}{c}{ Dislocation dissociation distance $(a)$} \\
& Basal $\langle\boldsymbol{a}\rangle$ & \multicolumn{2}{c}{ Prismatic $\langle\boldsymbol{a}\rangle$} & \multicolumn{2}{c}{ Pyramidal I $\langle\boldsymbol{c}+\boldsymbol{a}\rangle$} & Pyramidal II $\langle\boldsymbol{c}+\boldsymbol{a}\rangle$ \\
& Edge & Screw & Edge & Screw & Edge & Screw & Edge & Screw \\
\hline Ab initio & 7.0 & 4.0 & 1.0 & 2.0 & & & 6.6 & 4.6 \\
MEAM & 12.5 & 4.0 & 1.0 & 1.0 & 8.3 & 5.3 & 6.4 & 4.2 \\
\hline
\end{tabular}

Table 3. Magnesium dislocation dissociation distance from ab initio and the MEAM potential. 

derived from it. The Version of Record is available online at http://dx.doi.org/10.1088/0965-0393/23/1/015004. Citation details: Wu, Z.; Francis, M. F.; Curtin, W. A. Model. Simul. Mater. Sci. Eng. 2015, 23 (1), 15004.

figure_6.pdf

Figure 6. Prismatic $\langle\boldsymbol{a}\rangle$ edge and screw dislocation core structures as visualized by the component of the Nye tensor and differential displacement plots. DFT results are replotted based on atom positions in Refs [13] and [46].

Figure 6 shows the $\langle\boldsymbol{a}\rangle$ edge and screw dislocations on prismatic planes. For the edge dislocation, the MEAM and DFT results are very similar, with a non-dissociated but broad core along the prismatic plane and no screw components, consistent with the GSF energy surface on the prismatic plane. For the screw dislocation, both MEAM and DFT results show pure screw components with no edge character. However, the MEAM potential shows a more compact core structure than predicted by the DFT. Close examination of the DD plots shows that the screw dislocation narrowly spreads its core into two partials in the DFT calculations while the core spreading is almost absent in the MEAM result. In MD simulations at 77 $\mathrm{K}$ (not shown), the MEAM dislocation core structure is stable, but occasionally transforms to a core structure similar to the DFT one. This suggests that the core structure shown by 
This is an author-created, un-copyedited version of an article accepted for publication/published in Modelling and Simulation in Materials Science and Engineering. IOP Publishing Ltd is not responsible for any errors or omissions in this version of the manuscript or any version derived from it. The Version of Record is available online at http://dx.doi.org/10.1088/0965-0393/23/1/015004. Citation details: Wu, Z.;

Francis, M. F.; Curtin, W. A. Model. Simul. Mater. Sci. Eng. 2015, 23 (1), 15004.

DFT calculations is metastable for the MEAM potential. At $300 \mathrm{~K}$, the MEAM dislocation core cannot be stabilized on the prismatic plane, cross-slipping onto the basal plane and dissociating into two partial dislocations as shown in figure 5. Calculations of the core energy of the screw dislocation on the prismatic and basal planes show that $E^{\text {prism }}(r)-E^{\text {basal }}(r) \approx$ $0.02 \mathrm{eV} / \AA$. This suggests that (i) the screw dislocation on prismatic plane is metastable with respect to that on basal plane and (ii) the energy difference per $\AA$ is comparable to the thermal energy at room temperature so that thermally-activated cross-slip at moderate temperatures is possible, as observed in our finite temperature MD simulations, although the exact energy barrier for cross-slip is not calculated here.

figure_7.pdf

Figure 7. Pyramidal I $\langle\boldsymbol{c}+\boldsymbol{a}\rangle$ edge and screw dislocation core structures as visualized by the component of the Nye tensor and differential displacement plots.

Figure 7 shows the $\langle\boldsymbol{c}+\boldsymbol{a}\rangle$ edge and screw dislocation core structures on pyramidal I planes. No DFT calculations are currently available for these two dislocations. The MEAM potential predicts both dislocations to dissociate into partials of $1 / 2\langle\boldsymbol{c}+\boldsymbol{a}\rangle$. These partial dislocations are primarily pure edge or pure screw dislocations. Close examination shows that the partial core structures do differ, particularly in the case of the screw dislocation. This is not surprising given the differences in the GSF energy surface along the $\langle\boldsymbol{c}+\boldsymbol{a}\rangle$ direction. As shown in figure 7 , the screw partial dislocation on the left exhibits minor core spreading along the prismatic plane while the partial on the right has its core spreading along the pyramidal I plane. The differences in the core structure of the two partials may result in asymmetrical Peierls barriers and slip behavior.

Figure 8 shows the $\langle\boldsymbol{c}+\boldsymbol{a}\rangle$ edge and screw dislocation core structures on pyramidal II planes as predicted by the MEAM potential and DFT. Similar to the pyramidal I case, the 

derived from it. The Version of Record is available online at http://dx.doi.org/10.1088/0965-0393/23/1/015004. Citation details: Wu, Z.; Francis, M. F.; Curtin, W. A. Model. Simul. Mater. Sci. Eng. 2015, 23 (1), 15004.

figure_8.pdf

Figure 8. Pyramidal II $\langle\boldsymbol{c}+\boldsymbol{a}\rangle$ edge and screw dislocation core structures as visualized by the component of the Nye tensor and differential displacement plots. DFT results are replotted based on atom positions in Ref [15].

MEAM potential predicts both dislocations to dissociate into partials of $1 / 2\langle\boldsymbol{c}+\boldsymbol{a}\rangle$ with the partial dislocations having primarily pure edge or pure screw character and different partial core structures. For the edge dislocation, the MEAM and DFT results show very similar dislocation core structures. For the screw dislocation, the MEAM result exhibits some core spreading along the prismatic plane while the DFT result shows a core structure localized along the pyramidal II plane. Overall, the MEAM potential retains the general structures of the DFT dislocation cores.

The $\langle\boldsymbol{c}+\boldsymbol{a}\rangle$ screw dislocations have marginally lower core energy $(0.004 \mathrm{eV} / \AA ̊)$ on the pyramidal II plane as compared to the pyramidal I plane. MD simulations show that the $\langle\boldsymbol{c}+\boldsymbol{a}\rangle$ screw dislocation on the pyramidal I plane is stable at $77 \mathrm{~K}$ but cross-slips onto the 
This is an author-created, un-copyedited version of an article accepted for publication/published in Modelling and Simulation in Materials Science and Engineering. IOP Publishing Ltd is not responsible for any errors or omissions in this version of the manuscript or any version derived from it. The Version of Record is available online at http://dx.doi.org/10.1088/0965-0393/23/1/015004. Citation details: Wu, Z.;

Francis, M. F.; Curtin, W. A. Model. Simul. Mater. Sci. Eng. 2015, 23 (1), 15004.

pyramidal II plane at $300 \mathrm{~K}$. At $300 \mathrm{~K}$, the screw dislocation predominantly remains on the pyramidal II plane but occasionally cross-slips back to the pyramidal I plane. The $\langle\boldsymbol{c}+\boldsymbol{a}\rangle$ edge dislocation on the pyramidal I plane is intriguing because its line direction is not a rational direction of the crystal lattice. Nevertheless, the edge dislocation on the pyramidal I plane has a marginally lower core energy $(0.005 \mathrm{eV} / \AA)$ than that on the pyramidal II plane. The elastic energy of the pyramidal I edge also increases more slowly with radial distance around the core than does the elastic energy of the dislocation on the pyramidal II plane. Comparing the energies between the $\langle\boldsymbol{c}+\boldsymbol{a}\rangle$ edge and screw dislocations, the screw dislocations have both lower (estimated) core energy and slower increase of elastic energy with radial distance than their edge counterparts, making the screw dislocations more energetically favorable. This then further suggests that slip is preferred on the pyramidal II plane, since it has the lower screw dislocation energy.

figure_9.pdf

Figure 9. $\{10 \overline{1} 2\}\langle\overline{1} 011\rangle$ tensile twinning edge and screw dislocation structures.

Figure 9 shows the atomic structures of the edge and screw $\{10 \overline{1} 2\}\langle\overline{1} 011\rangle$ tensile twinning dislocations. Because of the atomic shuffling process involved in the migration of the twinning dislocation and the small Burgers vector, it is difficult to make direct comparisons between MEAM and DFT results using either components of the Nye tensor and or DD plots. We therefore superimposed the atom positions predicted by the MEAM and DFT, as shown in figure 9, for the edge dislocation. The close match of atom positions shows that the MEAM potential produces the twinning interface and twinning dislocation core structure in remarkable agreement with DFT. There is no DFT result for the screw twinning dislocation.

Having examined the various dislocation core structures predicted by the current MEAM potential, we now consider their Peierls stresses. Table 4 summarizes the Peierls stress computed at $0 \mathrm{~K}, 77 \mathrm{~K}$ and $300 \mathrm{~K}$, together with available experimental measurements. A few important observations can be made. First, in the case of finite Peierls stresses, screw dislocations exhibit higher Peierls stress than their edge dislocation counterparts at all temperatures. The magnitudes of the Peierls stresses on the basal, prismatic, and pyramidal I and II planes are in the same order as their GSF energies. Second, all dislocations on the pyramidal I, II and twinning interfaces show clear, asymmetrical Peierls stresses at 0 $\mathrm{K}$, consistent with the asymmetrical core structures of the dissociated dislocations. Third, 
This is an author-created, un-copyedited version of an article accepted for publication/published in Modelling and Simulation in Materials Science and Engineering. IOP Publishing Ltd is not responsible for any errors or omissions in this version of the manuscript or any version derived from it. The Version of Record is available online at http://dx.doi.org/10.1088/0965-0393/23/1/015004. Citation details: Wu, Z.;

Francis, M. F.; Curtin, W. A. Model. Simul. Mater. Sci. Eng. 2015, 23 (1), 15004.

\begin{tabular}{|c|c|c|c|c|c|c|c|c|c|c|}
\hline \multicolumn{11}{|c|}{ Peierls stress (MPa) } \\
\hline & \multicolumn{2}{|c|}{$\operatorname{Basal}\langle\boldsymbol{a}\rangle$} & \multicolumn{2}{|c|}{ Prismatic $\langle\boldsymbol{a}\rangle$} & \multicolumn{2}{|c|}{ Pyramidal I $\langle\boldsymbol{c}+\boldsymbol{a}\rangle$} & \multicolumn{2}{|c|}{ Pyramidal II $\langle\boldsymbol{c}+\boldsymbol{a}\rangle$} & \multicolumn{2}{|c|}{$10 \overline{1} 2$}$\langle\overline{1} 011\rangle$ twin \\
\hline & Edge & Screw & Edge & Screw & Edge & Screw & Edge & Screw & Edge & Screw \\
\hline $\operatorname{Exp}(\sim 300 \mathrm{~K})$ & & $5^{a}$ & & $40^{a}$ & & & 4 & $0^{a}$ & & \\
\hline MEAM (0K) & 0.75 & 50 & 9.3 & 119 & $35(65)$ & $155(344)$ & 132 (159) & $331(598)$ & $71(93)$ & $134(136)$ \\
\hline MEAM $(77 \mathrm{~K})$ & 0 & 3 & 0 & 23 & $6.9(9.4)$ & $118(300)$ & $57 \quad(59)$ & $141(285)$ & 0 & 0 \\
\hline MEAM $(300 \mathrm{~K})$ & 0 & 0 & 0 & CSTBP & $6.3(7.8)$ & $35 \quad(75)$ & $7.2(10)$ & 45 (105) & 0 & 0 \\
\hline
\end{tabular}

Table 4. Peierls stress measurements from experiments and the MEAM potential. At $300 \mathrm{~K}$, the prismatic $\langle\boldsymbol{a}\rangle$ screw dislocation cross-slips to basal plane (CSTBP). Numbers in parentheses are Peierls stresses in opposite directions.

${ }^{a}$ References [47]

the Peierls stresses drop quickly with increasing temperature. This is particularly true for dislocations with small Burgers vectors, like the twinning dislocations and basal plane dislocations. Finally, at $300 \mathrm{~K}$, the MEAM potential results are consistent with available experimental values. We emphasize that accurate capturing of Peierls stresses is a particular challenge, and that the main goal is to have potentials that predict the correct ordering among different slip systems and with reasonable agreement with experiments.

\section{Fracture Properties}

In comparatively brittle materials like $\mathrm{Mg}$, fracture by crack propagation can be more relevant than in more-ductile metals. In all systems, the crack-tip competition between cleavage and dislocation emission plays an important role since dislocation emission blunts the crack, increasing the necessary applied loads to drive fracture, which enables plastic dissipation away from the crack to provide additional toughening. Therefore, the fracture properties of the MEAM potential must be validated if it is to be used for meaningful studies of fracture.

Here, we study the crack tip behavior of two atomically-sharp basal plane cracks loaded under mode I crack tip stress intensity factor $K_{I}$. Fracture mechanics theory predicts the critical stress intensity factor for Griffith cleavage [48] to be

$$
K_{I c}=\sqrt{\frac{G}{A}}
$$

where $G=2 \gamma_{s}$ is the energy release rate and

$$
A=\sqrt{\frac{a_{11} a_{22}}{2}} \sqrt{\frac{2 a_{12}+a_{66}}{2 a_{11}}+\sqrt{\frac{a_{22}}{a_{11}}}}
$$

and $a_{i j}$ are the elastic compliance constants for the plane-strain stress state. The surface energy $\gamma_{s}$ thus determines brittle fracture, as is well-known. The critical stress intensity factor for dislocation emission is computed based on the theory of Rice [49] as

$$
K_{\text {Ie }}=f_{I}^{-1}(\theta) \sqrt{\frac{2 \mu}{1-\nu}\left[1+(1-\nu) \tan ^{2} \phi\right] \gamma_{u s}}
$$


This is an author-created, un-copyedited version of an article accepted for publication/published in Modelling and Simulation in Materials Science and Engineering. IOP Publishing Ltd is not responsible for any errors or omissions in this version of the manuscript or any version derived from it. The Version of Record is available online at http://dx.doi.org/10.1088/0965-0393/23/1/015004. Citation details: Wu, Z.; Francis, M. F.; Curtin, W. A. Model. Simul. Mater. Sci. Eng. 2015, 23 (1), 15004.

where $f_{I}(\theta)=\cos ^{2}(\theta / 2) \sin (\theta / 2), \theta$ is the angle between the crack plane and slip plane, $\phi$ is the angle between the dislocation Burgers vector and crack front direction in the slip plane, $\mu$ is an appropriate shear modulus, $\nu$ is the Poisson ratio, and $\gamma_{u s}$ is the unstable stacking fault energy. The competition between brittle cleavage and blunting by dislocation emission thus depends primarily on the surface and unstable stacking fault energies, with additional factors of elasticity and crystallography entering.

Applying the above analysis to basal cracks in $\mathrm{Mg}$, we use the material parameters computed for the new MEAM potential, which agree well with DFT and/or experiments, to compute the critical stress intensities for fracture by cleavage and dislocation emissions. For cleavage on the basal plane, the critical stress intensity is $K_{I c}=0.255 \mathrm{MPa} \mathrm{m}^{1 / 2}$ as given by equation (3), independent of the orientation of the crack front. For dislocation emission, we consider dislocation emission on the slip planes that intersect the crack front along the entire crack line. For the orientation I basal plane crack, i.e., a crack line along [1릴, the relevant slip plane is associated with dislocation emission on the pyramidal I plane. For the orientation II basal plane crack, i.e., a crack line along [1010], the relevant slip plane is associated with dislocation emission along the pyramidal II plane. To calculate $K_{I e}$ for the two orientations analyzed here, we use the shear modulus $\mu$ resolved along the slip direction in the plane of interest, as obtained by rotating the stiffness tensor into coordinate systems aligned with such slip systems. The GSF energy plot and the various dissociated dislocation core structures shown earlier suggest that nucleation of partial dislocations would occur first in preference to full dislocations. Therefore, we take the unstable stacking energy corresponding to the nucleation of the first partial dislocation as $\gamma_{u s}$ in Equation (5). The unstable stacking fault energy for the first $\langle\boldsymbol{c}+\boldsymbol{a}\rangle$ partial dislocation on the Pyramidal I plane is $\gamma_{u s}=0.319 \mathrm{~J} / \mathrm{m}^{2}$ and the stress intensity for dislocation emission in orientation I is predicted to be $K_{I e}=0.345 \mathrm{MPa}$ $\mathrm{m}^{1 / 2}$, while for pyramidal II plane, the unstable stacking fault energy for the first $\langle\boldsymbol{c}+\boldsymbol{a}\rangle$ partial dislocation is $\gamma_{u s}=0.295 \mathrm{~J} / \mathrm{m}^{2}$, and the predicted stress intensity for dislocation emission in orientation II is $K_{I e}=0.353 \mathrm{MPa} \mathrm{m}^{1 / 2}$. Both values of $K_{I e}$ are rather larger than $K_{I c}$, so that basal cleavage is expected to occur without any dislocation emission.

Figure 10 shows a cross-section of the atomistic crack tip region in a plane perpendicular to the crack line at various applied $K_{I}$ values. For both crack orientations, part (a) of the figure shows the initial crack tip structures determined from the anisotropic elastic solution at $K_{I}=0.98 K_{I c}$. Upon relaxation of the atomic positions with the far-field boundary $K$ field held fixed, part (b) of the figure shows that the crack closes, i.e. the crack tip moves towards left. Crack closure at a load slightly below the Griffith cleavage load is consistent with fracture theory for a material with almost no lattice trapping. When the initial crack is created at a load level above the Griffith cleavage value $\left(K_{I}=1.06 K_{I c}\right)$, part (c) of the figure shows that both cracks advance forward in a cleavage fracture mode, again fully consistent with a fracture theory for a material with almost no lattice trapping. As expected based on the theory, no dislocation emission is observed in either case for a range of $K_{I}$ values around $K_{I c}$; only cleavage is observed when $K_{I}>K_{I c}$. The current MEAM potential gives fracture behavior in very good agreement with continuum theory prediction. 
figure_10.pdf

Figure 10. Cross-sectional views of the (0001)[12̄10] and (0001)[1010] sharp cracks on basal planes. (a) The initial crack tip structures determined from the anisotropic elastic solution at $K_{I}=0.98 K_{I c}$, where $K_{I c}$ is the critical stress intensity for Griffith cleavage. (b) Relaxation of (a) with far-field boundary $K$ field held fixed. (c) Relaxed crack tip structures at $K_{I}=1.06 K_{I c}$.

\section{Discussion and conclusions}

We have presented detailed analyses of the GSF energies, dislocation core structures, Peierls barriers and fracture properties of a new MEAM potential for Mg. Overall, the MEAM potential shows satisfactory results in all the tests when compared to available DFT, continuum theory and experimental results. The original Kim potential has similar properties aside from the fracture properties, but all of the properties here had not been previously studied.

In the study of dislocation core structures, the $\langle\boldsymbol{a}\rangle$ edge dislocation on basal plane in MEAM has a wider dissociation separation as compared to that in DFT, despite both having similar partial core structures. The partial separation depends mainly on the stable stacking fault energy and slightly on the Peierls stresses of the partials. Since the basal plane stable stacking fault energy in MEAM is lower than that of the current DFT benchmark, it is not surprising that wider separation is seen in MEAM. The relatively lower stacking fault energy in MEAM is a result of the compromise we made during the fitting of the potential. In fact, we 
This is an author-created, un-copyedited version of an article accepted for publication/published in Modelling and Simulation in Materials Science and Engineering. IOP Publishing Ltd is not responsible for any errors or omissions in this version of the manuscript or any version derived from it. The Version of Record is available online at http://dx.doi.org/10.1088/0965-0393/23/1/015004. Citation details: Wu, Z.; Francis, M. F.; Curtin, W. A. Model. Simul. Mater. Sci. Eng. 2015, 23 (1), 15004.

can fit the potential to reproduce both the stacking fault energy and partial separations identical to the current DFT benchmark value. However, that results in a slightly lower $c / a$ ratio. Since $c / a$ ratio is very important for twinning deformation and the stacking fault energy values vary between different DFT calculations and supercell sizes [41], we choose to reproduce the $c / a$ ratio exactly as measured from experiments at the expense of lower stacking fault energy. The effects on glide due to wider partial separation are minimal to any physically interesting behavior, as the Peierls stress is effectively zero at any finite temperature. However, it may affect the nucleation of the trailing partial dislocations under certain conditions. For example, at crack tips, the critical stress intensity factor for nucleating the trailing partial depends on the equilibrium distance of the leading partial away from crack tips. Nevertheless, we expect the effect is not strong as the difference of the separation distance between MEAM and DFT is less than $2 \mathrm{~nm}$.

The $\langle\boldsymbol{a}\rangle$ screw dislocation on basal plane dissociates into two partials with both edge and screw components, making its cross-slip relatively difficult as it involves constriction of the two partials or nucleation of stair-rod dislocations. In contrast, the $\langle\boldsymbol{a}\rangle$ screw dislocation on the prismatic plane and $\langle\boldsymbol{c}+\boldsymbol{a}\rangle$ screw dislocation on the pyramidal I and II planes dissociate into nearly pure screw partial dislocations, which makes their cross-slips readily achievable through cross-slip by individual partial dislocation. As a result, such cross-slip is directly observable at the MD time scales. The $\langle\boldsymbol{c}+\boldsymbol{a}\rangle$ screw dislocations on the pyramidal I and II plane have nearly equal dislocation energy. At room temperatures, these dislocations primarily stay on the pyramidal II plane. This might be correlated to the experimental observation that $\langle\boldsymbol{c}+\boldsymbol{a}\rangle$ dislocations are often seen on pyramidal II planes. The line direction of $\langle\boldsymbol{c}+\boldsymbol{a}\rangle$ edge dislocation on the pyramidal I plane is not a rational direction of the crystal lattice and therefore we expect this dislocation to adopt a mixed-character dislocation line orientation. The GSF energy curve also suggests a complex MEP deviating from the $\langle\boldsymbol{c}+\boldsymbol{a}\rangle$ Burgers vector direction. This makes the nucleation and gliding of this dislocation intriguing. Whether and how this dislocation can be nucleated are not clear at present and require further study.

In the fracture tests, because of the high stacking fault energy on pyramidal I and II planes, cleavage is the preferred mode of deformation at atomically-sharp basal plane cracks. This intrinsically brittle behavior will have important implications for understanding fracture behavior, fracture toughness, and fatigue crack growth in $\mathrm{Mg}$ and its alloys, and will be the subject of our future work. In this paper devoted to validation of the new MEAM potential, we only focus on the facts that the potential predicts brittle cleavage (i) as expected based on theory using material parameters that are well-predicted by the potential and (ii) with almost zero lattice trapping as expected for most metals. As discussed in Section 3, these important features were not predicted using the Kim potential. In addition, both DFT and MEAM show that the surface energies of the different low-index surfaces are very similar. This indicates $\mathrm{Mg}$ can potentially cleave along different crystallographic planes. As we have shown here, atomically-sharp basal plane cracks advance near the Griffith cleavage loading. This behavior may change if the sharp crack is oriented along pyramidal or twinning planes, as dislocation emission along basal planes is relatively easier and may occur before reaching 
This is an author-created, un-copyedited version of an article accepted for publication/published in Modelling and Simulation in Materials Science and Engineering. IOP Publishing Ltd is not responsible for any errors or omissions in this version of the manuscript or any version derived from it. The Version of Record is available online at http://dx.doi.org/10.1088/0965-0393/23/1/015004. Citation details: Wu, Z.;

Francis, M. F.; Curtin, W. A. Model. Simul. Mater. Sci. Eng. 2015, 23 (1), 15004.

Griffith cleavage values. More interestingly, blunted cracks may favor dislocation emission more than cleavage. All these would require further investigations.

It has been a challenging task for empirical and semi-empirical interatomic potentials of $\mathrm{Mg}$ to reproduce its various dislocation core structures, as all other potentials that have been examined fully for dislocation cores give poor descriptions for some cases [13, 45, 15]. The current potential using the MEAM formulation shows distinct advantages over previous EAM type potentials in reproducing the dislocation core and other mechanical properties. There are also many other potentials for Mg (e.g., Refs. [50, 51]) that have been fit to its bulk properties and some GSF energies, but these have not been tested for dislocations. Furthermore, our current and previous results [45] show that GSF energy alone is not sufficient to get good dislocation properties and therefore, their validity remains unclear in study focusing on dislocations and plasticity.

In summary, we have developed an MEAM potential for magnesium based on the work of Kim et al [18]. Through extensive testing and validation against existing DFT, continuum theory and experimental results, we show that this new MEAM potential reproduces all the necessary properties governing deformation, including the various GSF energies, dislocation core structures, Peierls stresses, surface energies and basal plane cohesive strength. As a result, the MEAM potential can completely describe all the important slip systems of $\mathrm{Mg}$. This is in stark contrast to previous EAM potentials which can only reproduce some of these properties. Our results further suggest that the present MEAM formulation can be a robust empirical interatomic potential for modelling general $\mathrm{Mg}$ deformation and fracture behavior in future.

\section{Acknowledgments}

The authors thank D. R. Trinkle and M. Ghazisaeidi for sharing the DFT data on dislocation core structures. ZW gratefully acknowledges the financial support from the Agency for Science, Technology and Research (A*STAR), Singapore. WAC acknowledges support of this work through a European Research Council Advanced Grant, "Predictive Computational Metallurgy", ERC Grant agreement No. 339081 - PreCoMet.

\section{References}

[1] Pollock T M 2010 Science 328 986-987

[2] Hirth J P and Lothe J 1982 Theory of Dislocations 2nd ed (John Wiley \& Sons)

[3] Li B and Ma E 2009 Acta Materialia 57 1734-1743

[4] Yu Q, Qi L, Chen K, Mishra R K, Li J and Minor A M 2012 Nano Letters 12 887-892

[5] Syed B, Geng J, Mishra R K and Kumar K S 2012 Scripta Materialia 67 700-703

[6] El Kadiri H, Kapil J, Oppedal A L, Hector Jr L G, Agnew S R, Cherkaoui M and Vogel S C 2013 Acta Materialia 61 3549-3563

[7] Yu Q, Wang J and Jiang Y 2013 Journal of Materials 2013903786

[8] Kaushik V, Narasimhan R and Mishra R K 2014 Materials Science and Engineering: A 590 174-185

[9] Liu B Y, Wang J, Li B, Lu L, Zhang X Y, Shan Z W, Li J, Jia C L, Sun J and Ma E 2014 Nat Commun 5 3297 
This is an author-created, un-copyedited version of an article accepted for publication/published in Modelling and Simulation in Materials Science and Engineering. IOP Publishing Ltd is not responsible for any errors or omissions in this version of the manuscript or any version derived from it. The Version of Record is available online at http://dx.doi.org/10.1088/0965-0393/23/1/015004. Citation details: Wu, Z.;

Francis, M. F.; Curtin, W. A. Model. Simul. Mater. Sci. Eng. 2015, 23 (1), 15004.

Magnesium interatomic potential for simulating plasticity and fracture phenomena

[10] Daw M S and Baskes M I 1984 Physical Review B 29 6443-6453

[11] Liu X Y, Adams J B, Ercolessi F and Moriarty J A 1996 Modelling and Simulation in Materials Science and Engineering 4293

[12] Sun D Y, Mendelev M I, Becker C A, Kudin K, Haxhimali T, Asta M, Hoyt J J, Karma A and Srolovitz D J 2006 Physical Review B 73 024116-12

[13] Yasi J A, Nogaret T, Trinkle D R, Qi Y, Jr L G H and Curtin W A 2009 Modelling and Simulation in Materials Science and Engineering 17055012

[14] Shin I and Carter E A 2012 Modelling and Simulation in Materials Science and Engineering 20015006

[15] Ghazisaeidi M, Hector Jr L G and Curtin W A 2014 Scripta Materialia 75 42-45

[16] Baskes M I 1992 Physical Review B 46 2727-2742

[17] Lee B J and Baskes M I 2000 Physical Review B 62 8564-8567

[18] Kim Y M, Kim N J and Lee B J 2009 Calphad 33 650-657

[19] Hartley C S and Mishin Y 2005 Acta Materialia 53 1313-1321

[20] Vitek V 1974 Crystalline Lattice Defects 5 1-34

[21] Nosé S 1984 Molecular Physics: An International Journal at the Interface Between Chemistry and Physics 52 255-268

[22] Nosé S 1984 Journal of Chemical Physics 81 511-519

[23] Hoover W G 1986 Physical Review A 34 2499-2500

[24] Melchionna S, Ciccotti G and Holian B L 1993 Molecular Physics: An International Journal at the Interface Between Chemistry and Physics 78 533-544

[25] Sun C T and Jin Z H 2011 Fracture Mechanics 1st ed (Academic Press)

[26] Plimpton S 1995 Journal of Computational Physics 117 1-19 ISSN 0021-9991

[27] Kresse G and Furthmüller J 1996 Computational Materials Science 6 15-50

[28] Kresse G and Furthmüller J 1996 Physical Review B 54 11169-11186

[29] Blöchl P E 1994 Physical Review B 50 17953-17979

[30] Monkhorst H J and Pack J D 1976 Physical Review B 13 5188-5192

[31] Wang Y and Perdew J P 1991 Physical Review B 43 8911-8916

[32] Perdew J P, Chevary J A, Vosko S H, Jackson K A, Pederson M R, Singh D J and Fiolhais C 1992 Physical Review B 46 6671-6687

[33] Thomson R, Hsieh C and Rana V 1971 Journal of Applied Physics 42 3154-3160

[34] Curtin W A 1990 Journal of Materials Research 5 1549-1560

[35] Bernstein N and Hess D W 2003 Physical Review Letters 91 025501-4

[36] Zhu T, Li J and Yip S 2006 Proceedings of the Royal Society of London - A 462 1741-1761

[37] Wang J, Hirth J P and Tomé C N 2009 Acta Materialia 57 5521-5530

[38] Walker G B and Marezio M 1959 Acta Metallurgica 7 769-773

[39] Wachowicz E and Kiejna A 2001 Journal of Physics: Condensed Matter 1310767

[40] Simmons G and Wang H 1971 Single Crystal Elastic Constants and Calculated Aggregate Properties. A Handbook 2nd ed (The MIT Press)

[41] Wang Y, Chen L Q, Liu Z K and Mathaudhu S N 2010 Scripta Materialia 62 646-649

[42] Han J, Su X M, Jin Z H and Zhu Y T 2011 Scripta Materialia 64 693-696

[43] Smith A E 2007 Surface Science 601 5762-5765

[44] Tang J J, Yang X B, OuYang L, Zhu M and Zhao Y J 2014 Journal of Physics D: Applied Physics 47 115305

[45] Nogaret T, Curtin W A, Yasi J A, Hector Jr L G and Trinkle D R 2010 Acta Materialia 58 4332-4343

[46] Yasi J A, Hector Jr L G and Trinkle D R 2011 Acta Materialia $595652-5660$ ISSN 1359-6454

[47] Tonda H and Ando S 2002 Metallurgical and Materials Transactions A - Physical Metallurgy and Materials Science 33 831-836

[48] Griffith A A 1921 Philosophical Transactions of the Royal Society of London. Series A, Containing Papers of a Mathematical or Physical Character 221 163-198

[49] Rice J R 1992 Journal of the Mechanics and Physics of Solids 40 239-271

[50] Pei Z, Zhu L F, Friák M, Sandlöbes S, von Pezold J, Sheng H W, Race C P, Zaefferer S, Svendsen B, Raabe 
This is an author-created, un-copyedited version of an article accepted for publication/published in Modelling and Simulation in Materials Science and Engineering. IOP Publishing Ltd is not responsible for any errors or omissions in this version of the manuscript or any version derived from it. The Version of Record is available online at http://dx.doi.org/10.1088/0965-0393/23/1/015004. Citation details: Wu, Z.;

Francis, M. F.; Curtin, W. A. Model. Simul. Mater. Sci. Eng. 2015, 23 (1), 15004.

Magnesium interatomic potential for simulating plasticity and fracture phenomena

D and Neugebauer J 2013 New Journal of Physics 15043020

[51] Jelinek B, Groh S, Horstemeyer M F, Houze J, Kim S G, Wagner G J, Moitra A and Baskes M I 2012 Physical Review B $\mathbf{8 5} 245102-18$ 